\title{
Orthodontic treatment enhanced using PRP: An alternative to Orthognathic surgery?
}

\author{
Foltan $\mathrm{R}^{1}$, Petr $\mathrm{J}^{2}$, Streblov $\mathrm{J}^{3}$ and Guha $\mathrm{A}^{1,4^{*}}$ \\ ${ }^{1}$ Department of Oral and Maxillofacial Surgery and Institute of Clinical and Experimental Dental Medicine, 1st Faculty of Medicine and \\ General University Hospital, Charles University, Prague, Czech Republic. \\ ${ }^{2}$ PetrOrtho s.ro, Private practice, Prague, Czech Republic. \\ ${ }^{3}$ DK s.r.o, Private practice, Prague, Czech Republic. \\ ${ }^{4}$ Department of Otorhinolaryngology, 3rd Faculty of Medicine and University Hospital Kralovske Vinohrady, Charles University, Prague, \\ Czech Republic
}

Received: January 1, 2019; Accepted: February 05, 2019; Published: February 26, 2019

*Corresponding author: Dr.Anasuya Guha, Department of Otorhinolaryngology, 3rd Faculty of Medicine, University Hospital Kralovske Vinohrady, Charles University, Srobarova 1150/50, Prague, 10034, Czech Republic, Tel:+420267163171; Fax:+420267162330; Email: anasuya.guha@fnkv.cz

\begin{abstract}
Background: The aim was to use an alternative technique to orthognathic surgery with similar functional and aesthetic outcome with the added advantages of being less invasive and comfortable to a patient. Here, we evaluate the efficacy of PRP in the acceleration of Orthodontic treatment using Invisalign to correct a malocclusion of Class III with functional reverse bite in a patient who declined surgery but requested the least visible and time consuming procedure.

Methods: PRP was applied three times during the course of the therapy. The orthodontic treatment lasted 10 months. The patient was further provided with restorative care. Protrusion of teeth 11, 21 , and 22 by bodily movement, shaping of the upper dental arch and rectification of lower incisors were achieved with no adverse effect to the patient's dental health. Since we assumed that the pathophysiology of the effect of PRP is the same as for corticotomy, we must assume an effect lasting only three months. The great advantage of our method is the fact that it is a minimally invasive technique and hence we could repeat the procedure thrice.
\end{abstract}

Results: Three applications of PRP significantly shortened the length of orthodontic therapy from 21 months to 10 months.

Conclusions: We can conclude that although the technique is perhaps a bit lengthy in terms of time taken to achieve the desired results after surgery but it provides a safe alternative approach for patients who decline surgery or instances where surgery cannot be carried out.

Keywords: PRP; corticotomy; orthodontic; aligner; Invisalign;

\section{Introduction}

Most frequently, our patients complain about the length of the orthodontic treatment. The most time-consuming process is bone resorption by osteoclasts on the side of the root of the tooth where pressure force is applied. As far as we know, there is little possibility to increase their activity. We therefore have to focus on the inorganic bone compartment and methods which are able to reduce bone density. There are several techniques described which are mainly physical methods with similar principles namely vibrations, electrical current, pulsed electromagnetic field, low dose laser irradiation and so on. Most of these became obsolete due to lack of success rate and scientific evidence. The use of the RAP (Regional Acceleratory Phenomenon) or a certain form of distraction osteogenesis was supported by certain authors [1]. RAP is a phenomenon first described by Frost HM [2-3]. It is a rapid bone turnover occurring at the site of bone trauma and the adjacent bone. This time turnover is associated with higher vascularisation and cellularisation of bone and reversible local osteomalacia. Bell WH was probably the first to report this method in his study of osteotomy in monkeys, but did not use the term RAP [4]. The tooth movement is approximately three times faster in such affected bone. Modern literature describes a technique called corticotomy [5], based on bone traumatisation with a bur drill, such as peppering of cortical bone or local cortical osteotomy, often accompanied by bone augmentation procedures. Tooth movement is facilitated using RAP only. This is then called Periodontal Accelerated Osteogenic Orthodontics. Another technique uses RAP and cortical plate distraction osteogenesis with the aid of a piezo electric device, making more than $3 \mathrm{~mm}$ deep cortical incision, sometimes also lingual or labial side of the dental root, complemented with special osteotomy around the bone supporting the interdental papilla. The distraction and tooth movements are faster and can also be used to increase the volume of alveolar bone in cases of major discrepancy between tooth mass and the apical base. This technique is also called monocortical tooth dislocation and ligament distraction [6]. The bone trauma triggers the healing process. That is, it starts with the healing process cascade, activation and degranulation of thrombocytes and in particular, growth and chemotactic factors from the thrombocytes alpha granules incite processes in the bone which normally occur during a surgical trauma [2-3]. A third and more sensible method 
is to use local application of growth factors, such as prostaglandin E1 (PGE1) [7-9], since it is less invasive, therefore better tolerated by patients. Given that thrombocytes degranulation triggers the healing process, it seems logical that surgery could be avoided by direct subperiosteal application of platelets rich plasma (PRP). Presumably, it should have identical or similar pathophysiological effects, thus accelerating orthodontic therapy.

\section{Aims}

Our primary aim was to present the results of orthodontic treatment using Invisalign in combination with subperiosteal PRP in a 43-year old patient. Thereby, we wanted to demonstrate the efficacy and safety of this method. Moreover, we also endeavoured to show that PRP accelerates orthodontic treatment and this technique can be used as an alternative to Orthognathic surgery in certain cases.

\section{Case Description And Results}

A 43-year-old male patient was referred by his dentist to our clinic for orthodontic treatment. The dentist requested an orthodontic correction of tooth position and bite so that prosthetic, functional and aesthetic restoration could be done, to avoid pathological premature bite. Such cases cannot be treated by any long-term conservative or prosthetic therapy. A working diagnosis of class III malocclusion, functional reverse bite, and pathological teeth abrasion was made after clinical examination, dental impressions, the standard pretreatment series including intraoral (Figure 1) and extraoral photography as well as radiographs and cephalometric analysis (Figure 2). He was a non-smoker, with no significant medical and family history. There was no genetic predisposition.

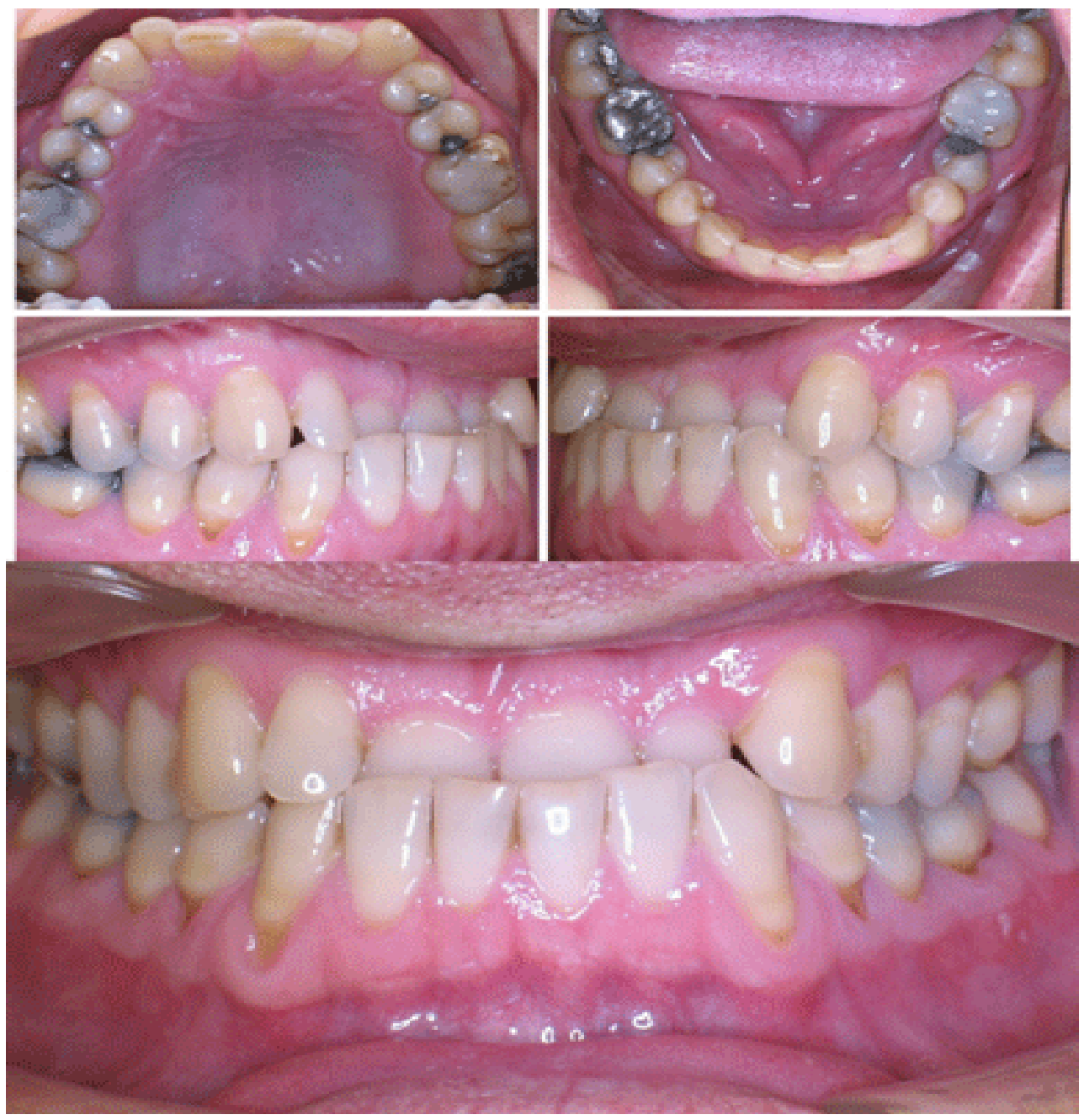

Figure 1: Pre-treatment series: Intraoral views: Upper occlusal (Upper left), Lower occlusal (Upper right), Right buccal in occlusion (Middle left) and Left buccal in occlusion (Middle Right) and Frontal - in occlusion (Lower) 


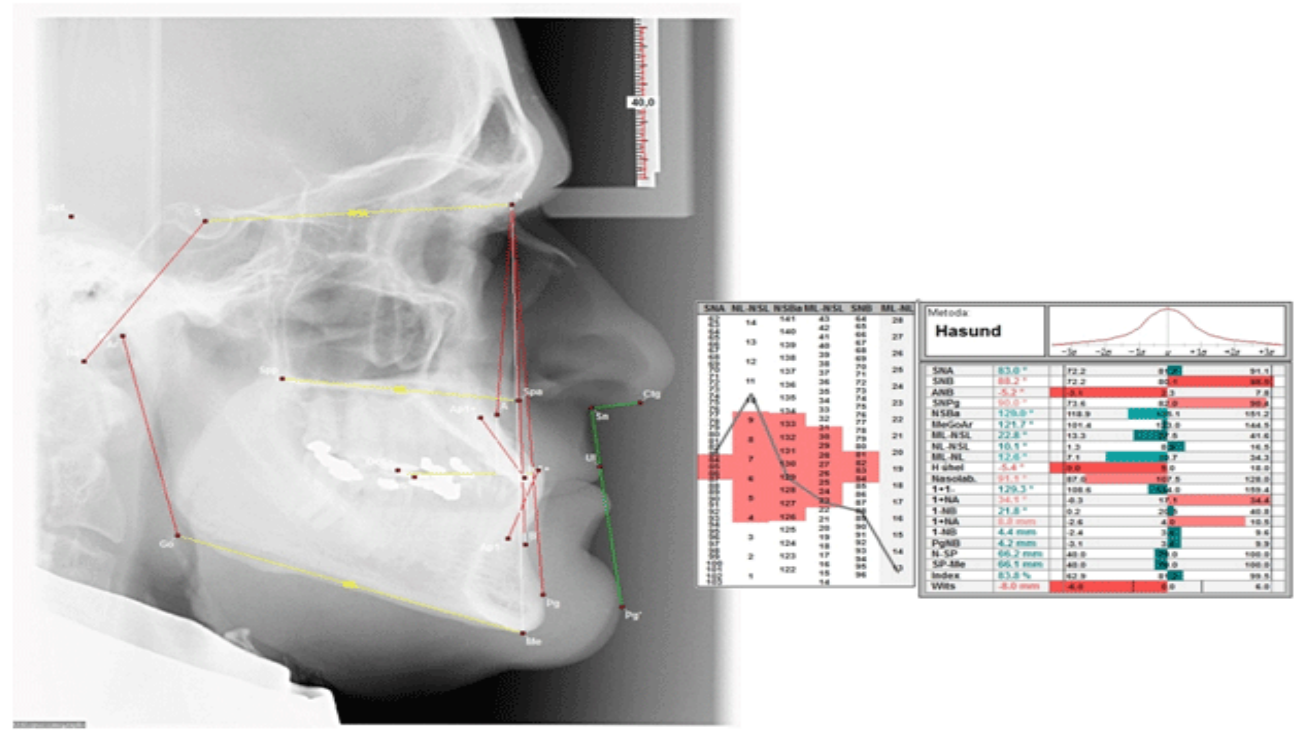

Figure 2: Cephalometric analysis

With clear treatment objectives such as correction of bite and aesthetic smile, following complete entry examination, 3 treatment alternatives were offered to the patient:

1. Orthognathic surgery - either using the surgery-first approach or the conventional combined technique with Orthodontic treatment - this was firmly turned down by the patient.

2. Orthodontic therapy alone - Invisalign $\AA$ installation protrusion of teeth 11,21 , and 22 , by bodily movement to maximum extent possible; shaping of the upper dental arch; rectification of lower incisors and their slight retraction. Creating conditions for functional and aesthetic restoration of the abraded upper anterior teeth and prosthetic bite rising.

3. Orthodontic therapy (as described above) with PRP application - in order to accelerate the treatment.

In view of the patient's age and choice, the least visible orthodontic method and fastest possible therapy (that is, the third option) had to be instituted.

PRP application included the use of Biomet Biologics set. We took $50 \mathrm{mls}$ of venous blood and after repeated centrifugation we obtained $2 \mathrm{mls}$ of PRP concentrate. Then after application of local anesthesia induced with Ubistesin forte ${ }^{\circledR}$, a thin needle was used to introduce PRP subperiosteally into the interdental space both labially and palatally. The application was very well tolerated by the patient. No perioperative or postoperative swelling was noted. The patient experienced minimum pain on the day of the application and none on the second day after application. This procedure was repeated three times during the therapy.
In addition, aesthetic and functional dentition restoration was also offered. For a minimalist version, direct composite restorations wax-up and silicone keys anteriorly and composite reconstruction of the posterior section could be utilized. In a direct procedure, it is very difficult to fine tune the optimal bite proportions and articulation. Optimal version of reconstruction constituted ceramic veneers and composite anteriorly or ceramic on lay in the posterior segments.

First application of plasma in the upper jaw was done, the following day treatment was initiated using the Invisalign $₫$ system with deployment of the first upper aligners. It was agreed with the patient that instead of the standard period of 14 days, the aligners would be replaced at an accelerated rate of every 5 days. Orthodontic review appointment showed that the patient had changed the aligners at the agreed accelerated rate; he experienced only a slight pressure after deployment of each successive aligner in the series; the aligners were fixed well. A month later, the upper aligner No. 12 was fixed, the treatment continued without problems.

Three months after first application of PRP, lower teeth stripping was carried out and initiation of treatment with Invisalign ${ }^{\circledR}$ of the lower dental arch continued at a standard speed (Figure 3). Concurrent deployment of the upper aligner No. 21 was done, accelerated replacement continued. Second application of plasma in the upper jaw was carried out at the end of the third month. And 3 weeks later, upper aligner No. 26 was fixed (Figure 4), the lower aligners were replaced at a standard rate. 


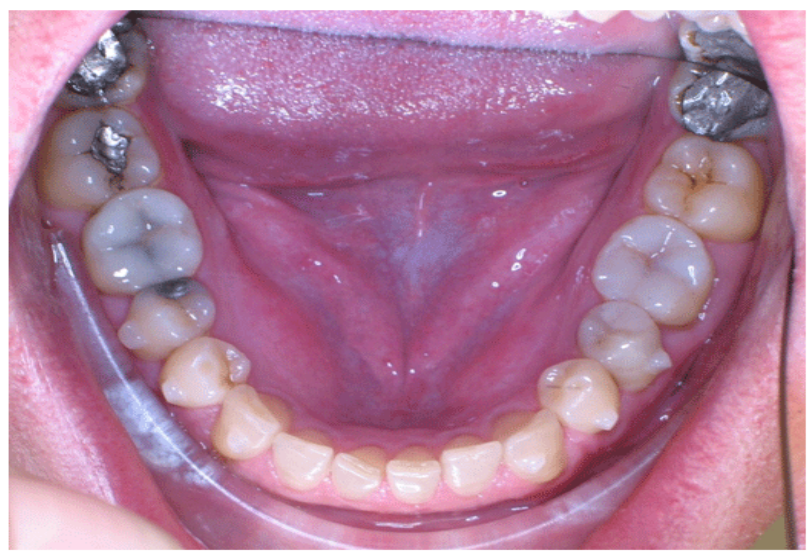

Figure 3: Lower teeth stripping and initiation of Invisalign of the lower dental arch

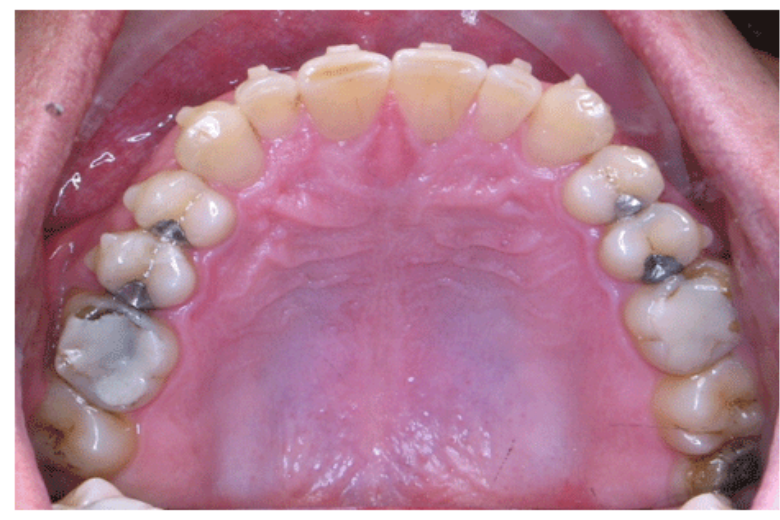

Figure 4: After second application of plasma and fixation of upper aligner No.26

The last aligners were placed approximately 5.5 months from initiation of orthodontic treatment. The shaping of the upper arch was good, but the achieved protrusion of the upper incisors was insufficient and still not allowing to perform the planned aesthetic and functional rehabilitation. A week later, the patient had a consultation with the Orthodontist. Orthodontic treatment continued as agreed. Dental impressions along with bite records were taken for "refinement" as part of the Invisalign $₫$ treatment.

Six months from the commencement of treatment, the third and last application of plasma was done in the upper jaw. The next day, upper "refinement" aligners were placed; from the start again, accelerated treatment with aligner replacement of 5 days was commenced. A further 3 months later, active orthodontic treatment using the Invisalign ${ }^{\circledR}$ system was terminated, documents were updated, retainer appliances (upper aesthetics Essix foil, upper retainer plate, lower fixed retainer and lower Essix) were applied. The orthodontic part of the treatment lasted 10 months.
On the 11th month, completion of aesthetic and functional restoration of the teeth was done. For aesthetic rehabilitation of the anterior part we used all-ceramic restoration because it is the most enduring way to achieve aesthetic results with colour and aesthetic unification of the most visible front teeth. Since the teeth are otherwise healthy, the most appropriate consideration was the least invasive solution. We fixed using a composite light solidifying material. Due to the minimum thickness of the ceramics, problems such as X-ray of solid ceramic reconstruction and achieving complete polymerization fixing were not encountered. Cushioning material can still affect the final colour reconstruction, in the present case, however, we chose the corresponding dentin shade, since teeth were not stained substantially from the structural point of view we tried to only add or strengthen enamel. Also in order to achieve harmonization of colour, the patient was also advised 3 weeks of tooth whitening prior to restoration. Final form of retainer appliances were inserted (Figure 5). Figure 6 shows the lateral view of the face and Figure 7 lateral skull X-rays pre-treatment and post-treatment. 


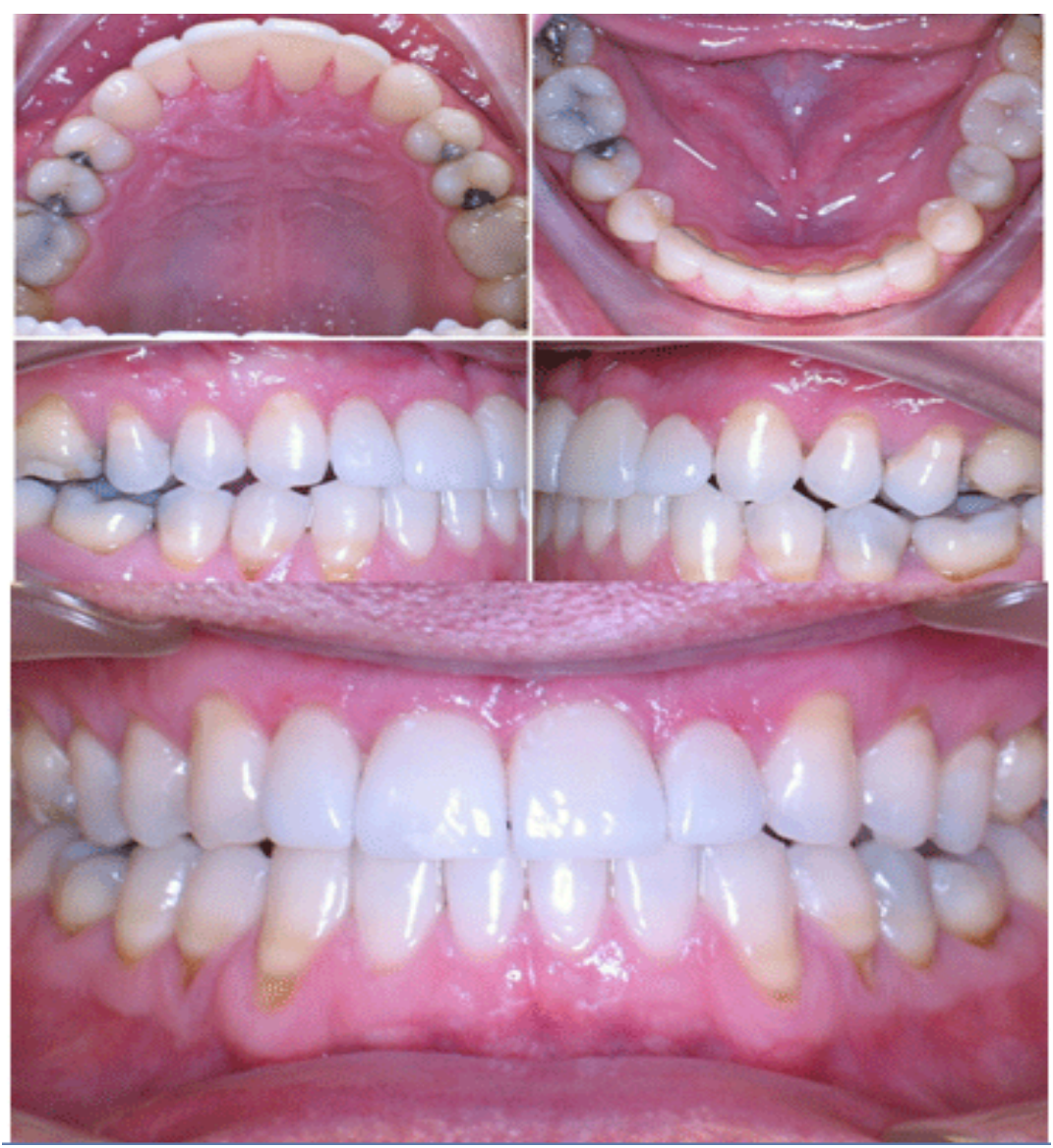

Figure 5: Post-treatment series: Intraoral views: Upper occlusal (Upper left), Lower occlusal (Upper right), Right buccal in occlusion (Middle left) and Left buccal in occlusion (Middle Right) and Frontal - in occlusion (Lower)
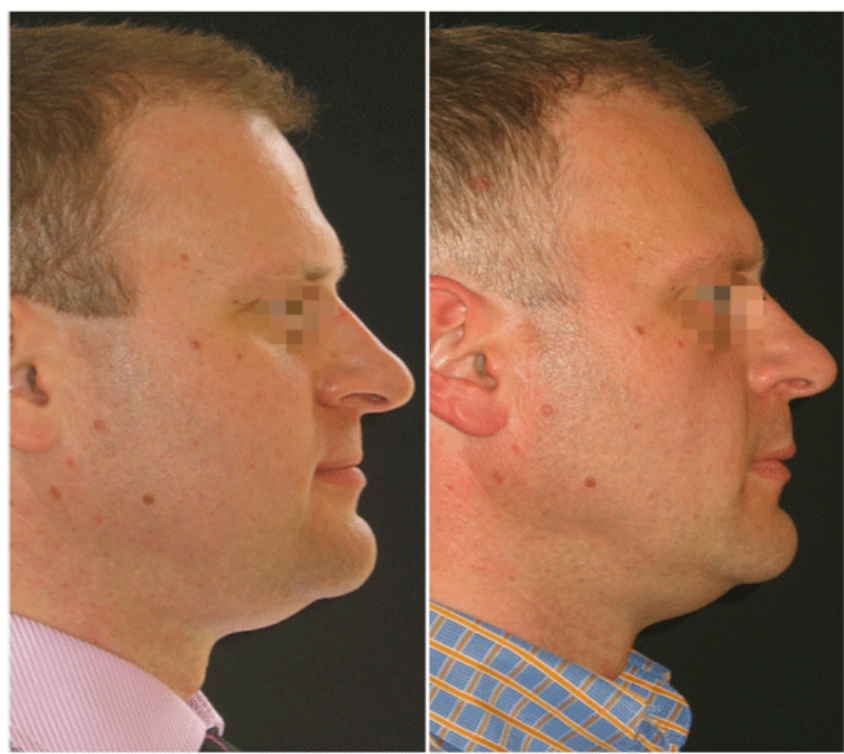

Figure 6: Lateral views of the face: Pre and post treatment 


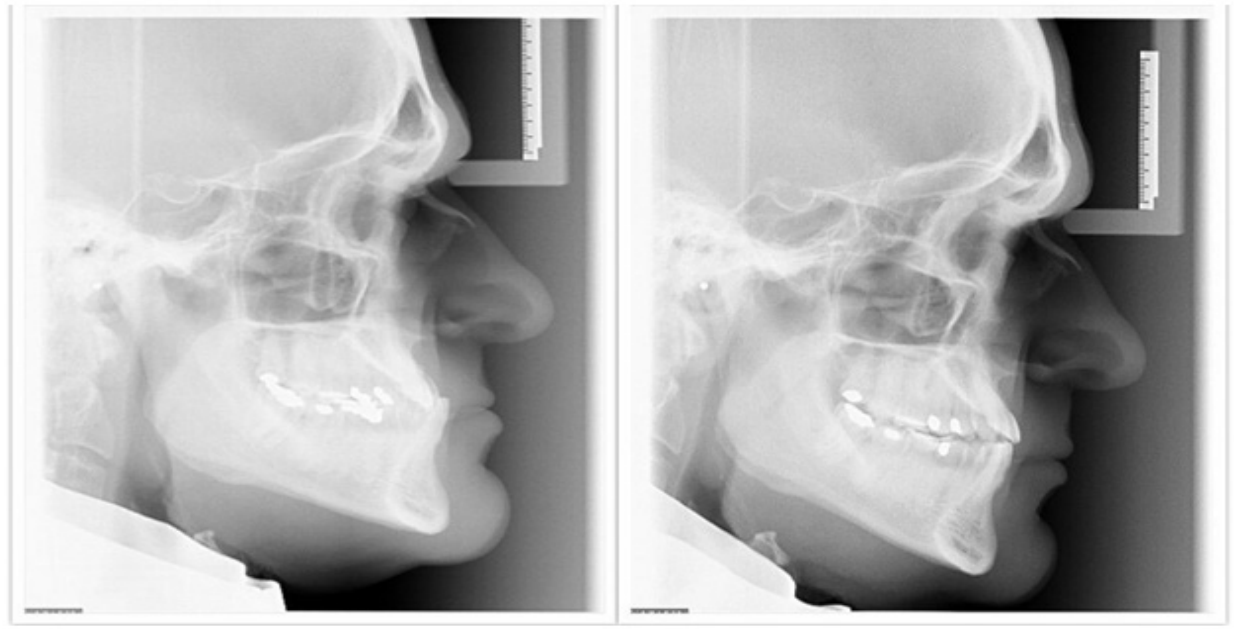

Figure 7: Lateral skull views: Pre and post treatment

\section{Discussion}

As far as we are aware, there has been no study published describing the combined use of PRP and Invisalign ${ }^{\circledR}$ for accelerated orthodontic therapy. In general, PRP is used mainly for bone augmentation procedures [10].

When using classic fixed appliances it is very difficult to define a "normal" duration of orthodontic therapy for individual patients, unless we conduct an individual split mouth study with the application of the active substance and placebo. In this respect, the Invisalign ${ }^{\circledR}$ method stands out due to clearly defined movements and relatively clearly defined duration of therapy. This is defined by the known number of aligners and their recommended replacement interval of 7 days. Faster replacement without the use of PRP or other more invasive methods is perceived by patients as extremely unpleasant and sometimes even impossible.

Because our described case is a borderline one, other therapy could be also used in this case, such as combined orthodonticsurgical therapy with Le Fort I advancement or corticotomy. However, in the absence of the patient's consent, the plans had to be discarded.

Studies discussing the topic of orthodontic therapy acceleration using Wilckodontics showed proven shortening of the therapy duration [5-11]. In our case study, the therapy lasted 10 months, although it would have taken 21 months if the standard Invisalign ${ }^{\circledR}$ treatment plan was followed. Since we assume that the pathophysiology of the effect of PRP is the same as for corticotomy, we must therefore presume an effect lasting only three months. The great advantage of our method is the fact that it is a minimally invasive technique, thus far less traumatising for the patient and hence we could repeat the procedure thrice.

The only two studies that found a significant increase in postoperative pain following corticotomy were those by Al-Naoum et al.[12] and Cassetta et al.[13], who had used more invasive surgical procedures. To the contrary, the study by Wilcko et al. shows that the thickness of the keratinised gingiva increases after this intervention [14]. Shoreibh et al. compared modified corticotomy-facilitated orthodontic tooth movement alone and modified corticotomy-facilitated orthodontic tooth movement combined with bone grafting, the incidence of apical root resorption and periodontal problems associated with orthodontic tooth movement were reduced. The incorporation of bone graft material significantly increased the alveolar bone density in adult patients [15]. However a systematic review, published last year, looking at studies utilising corticotomies to accelerate tooth movement during orthodontic treatment stated that even though the results of these studies included confirm that the combination approach reduces the duration of the treatment by accelerating tooth movement, however, few clinical trials have been conducted to date in this area, with small samples of patients and short-term follow-up, so the efficiency-safety ratio is not conclusive [16].

In our case, we did not see any complications even after repeated application of PRP and the patient did not suffer from any pain or limitations in normal life. It would also be interesting to monitor the quality of the alveolar bone using Cone Beam Computer Tomography and we are already working on such a study.

Studies investigating success rates of bone augmentation procedures using the PRP method are interesting. Although these techniques are generally recognised as beneficial, JovaniSancho et al. indicate in their review that only some of the PRP separation systems (Smart Prep, PRGF ARRANGEMENT) have been scientifically substantiated at least to some degree and even in such cases, only 33 studies, were able to document success of bone turnover procedures. For other systems, such as Curasan or Platelet Concentrate Collection System (PCCS), there has been no success rate of bone augmentation procedures documented in the available studies [17]. 
Other authors, such as Weibrich et al. compared the quality of the PRP concentrate obtained using the Platelet Concentrate Collection System (PCCS) and using the PRGF-kit. The PCCS method achieved higher concentration of TGF-beta1a PDGF-AB in the PRP. However, what appears to be much more interesting are the findings from this study, according to which, the concentrate contains approximately two times the number of leukocytes compared to normal values, whereas the PDGF kit is leukocytepoor. Leukocytes, being the main markers of inflammation, can have a significant impact on osteological processes [18], by increasing the quantity of growth factors and the duration of their release when applying PRP as shown by Dohan Ehrenfest et al [19]. Furthermore, accelerated orthodontic tooth movement was also supported by findings in animal studies. Gulec et al used histomorphometric analysis in rats, which showed decreased alveolar bone density by enhanced osteoclastic activity using both moderate and high concentrations of PRP [20]. Another study in dogs demonstrated significantly faster maxillary tooth movement with no obvious clinical or microscopic side effects [21].

\section{Conclusions}

Our case study shows that use of Invisalign $₫$ and three applications of PRP can significantly shorten the length of orthodontic therapy from 21 months to 10 months. Therefore, a combination of PRP application (a less-invasive technique) and Invisalign ${ }^{\circledR}$ (the least recognizable orthodontic appliance) has a clear logic and, from our experience, can lead to substantial acceleration of the orthodontic therapy. Although a larger cohort of patients is required to prove complete efficacy of this method, this patient can be considered as a pilot case, since the aim was achieved and the treatment proved successful. Furthermore, there is also the need to conduct long-term studies assessing orthodontic stability, three dimensional morphology of the alveolar bone as well as the exact pathophysiology of this process. To conclude, this technique not only proves to have an accelerated outcome, it achieves this in the least traumatic way. It is comfortable and acceptable to a patient. It can be considered where a patient declines surgery or some medical contraindication prohibits it. It also reduces the risks associated with surgery and general anesthesia therefore no recovery time is required. The added benefit of the technique is that it is safe to use and may be repeated according to requirement.

\section{References}

1. Shoreibah EA, Salama AE, Attia MS, Abu-Seida SM. Corticotomyfacilitated orthodontics in adults using a further modified technique. J Int Acad Periodontol. 2012 Oct;14(4):97-104.

2. Frost HM. The biology of fracture healing. An overview for clinicians. Part I. Clin Orthop Relat Res. 1989;(248):283-293.

3. Frost HM. The biology of fracture healing. An overview for clinicians. Part II. Clin Orthop Relat Res. 1989;(248):294-309.

4. Bell WH. Revascularization and bone healing after anterior maxillary osteotomy: a study using adult rhesus monkeys. J Oral Surg. 1969;27(4):249-255.
5. Wilco WM, Wilco T, Bouquot JE, Ferguson DJ. Rapid orthodontics with alveolar reshaping: two case reports of decrowding. Int J Periodontics Restorative Dent. 2001;21(1):9-19.

6. Vercellotti T, Podesta A. Orthodontic microsurgery: a new surgically guided technique for dental movement. Restorative Dent. 2007;27(4):325-331.

7. Yamasaki K, Shibata Y, Imai S, Tani Y, Shibasaki Y, Fukuhara T. Clinical application of prostaglandinE1 (PGE1) upon orthodontic tooth movement. Am J Orthod. 1984;85(6):508-518.

8. Patil AK, Keluskar KM, Gaitonde SD. The clinical application of PGE1 in orthodontic tooth movement. J Indian Orthod Soc 2005; 38: 91-98

9. Spielmann T, Weislander L, Hefti AF. Acceleration of orthodontically induced tooth movement through the local application of prostaglandin (PGE1). Schweizer Monatsschrift fur Zahnmedizin. 1989;99:162-116

10. Owen AH III. Accelerated Invisalign treatment. J Clin Orthod. 2001;35(6):381-385.

11. Wilcko WM, Ferguson DJ, Bouquot JE, Wilcko MT. Rapid orthodontic decrowding with alveolar augmentation: case report. World J Orthod. 2003;4(3):197-205.

12. Al-Naoum F, Hajeer MY, Al-Jundi A. Does alveolar corticotomy accelerate orthodontic tooth movement when retracting upper canines? A splitmouth design randomized controlled trial. J Oral Maxillofac Surg. 2014;72:1880-1889. doi: 10.1016/j.joms.2014.05.003

13. Cassetta M, Di Carlo S, Giansanti M, Pompa V, Pompa G, Barbato E. The impact of osteotomy technique for corticotomy-assisted orthodontic treatment (CAOT) on oral health-related quality of life. Eur Rev Med Pharmacol Sci. 2012;16(12):1735-1740.

14. Wilcko MT, Ferguson DJ, Makki L, Wilcko WM. Keratinized Gingiva Height Increases After Alveolar Corticotomy and Augmentation Bone Grafting. J Periodontol. 2015;86(10): 1107-15.

15. Shoreibah EA, Ibrahim SA, Attia MS, Diab MM. Clinical and radiographic evaluation of bone grafting in corticotomy-facilitated orthodontics in adults. J Int Acad Periodontol. 2012;14(4):105-13.

16. Fernandez-Ferrer L, Montiel-Company JM, Candel-Marti E, AlmerichSilla JM, Penarrocha-Diago M, Bellot-Arcis C. Corticotomies as a surgical procedure to accelerate tooth movement during orthodontic treatment: A systematic review. Med Oral Patol Oral Cir Bucal. 2016;21(6):e703-e712.

17. Jovani-Sancho MD, Sheth CC, Marqués-Mateo M, Puche-Torres M. Platelet-Rich Plasma: A Study of the Variables that May Influence Its Effect on Bone Regeneration.Clin Implant Dent Relat Res. 2015.

18. Weibrich G, Kleis WK, Hitzler WE, Hafner G. Comparison of the platelet concentrate collection system with the plasma-rich-in-growth-factors kit to produce platelet-rich plasma: a technical report. Int J Oral Maxillofac Implants. 2005;20(1):118-23.

19. Dohan Ehrenfest DM, Bielecki T, Jimbo R, Barbé G, Del Corso M and Inchingolo $\mathrm{F}$, et al. Do the fibrin architecture and leukocyte content influence the growth factor release of platelet concentrates? An vidence-based answer comparing a pure platelet-rich plasma (P-PRP) gel and a leukocyte- and platelet-rich fibrin (L-PRF). Curr Pharm Biotechnol. 2012;13(7):1145-1152. 
20. Gulec A, Bakkalbasi BC, Cumbul A, Uslu U, Alev B, Yarat A. Effects of local platelet-rich plasma injection on the rate of orthodontic tooth movement in a rat model: A histomorphometric study. Am J Orthod Dentofacial Orthop. 2017;151(1):92-104
21. Rashid A, ElSharaby FA, Naseef EM, Mehanni S, Mostafa YA. Effect of platelet-rich plasma on orthodontic tooth movement in dogs. Orthod Craniofac Res. 2017;20(2):102-110. doi: 10.1111/ocr.12146 